\title{
A Model to Assess Collaboration Performance in a Collaborative Business Ecosystem
}

\author{
Paula Graça ${ }^{\mathrm{a}, \mathrm{b}}$, Luís M. Camarinha-Matos ${ }^{\mathrm{a}}$ and Filipa Ferrada ${ }^{\mathrm{a}}$ \\ ${ }^{a}$ Faculty of Sciences and Technology and Uninova CTS, NOVA University of Lisbon, \\ Campus de Caparica, 2829-516 Caparica, Portugal \\ ${ }^{\mathrm{b}}$ Instituto Superior de Engenharia de Lisboa, Instituto Politécnico de Lisboa, \\ Rua Conselheiro Emídio Navarro 1, 1959-007 Lisbon, Portugal \\ mgraca@deetc.isel.pt, cam@uninova.pt, faf@uninova.pt
}

\begin{abstract}
In a Collaborative Business Ecosystem, Performance Indicators are a useful mechanism to assess collaboration performance, inducing selfadjustment in organization's profile, thereby improving the sustainability of the ecosystem. Using system dynamics and agent-based modelling, a simulation model is assembled to show organizations' self-adjustment by improving their profile in response to an assessment through a chosen set of performance indicators, such as innovation indicator, contribution indicator and prestige indicator. The natural reaction of organizations (similar to individuals) towards improving their performance according to the way they are evaluated, is modelled considering different enterprise profiles categorized into various classes of responsiveness, to better simulate the diversity in a real collaborative business ecosystem. Preliminary results of this approach are presented and discussed.
\end{abstract}

Keywords: Collaborative Networks, Business Ecosystem, Performance Indicators, System Dynamics, Agent Based Modelling.

\section{Introduction}

Business ecosystems are crossing a new era anchored in more interconnected and powerful digital platforms, following the dynamics and trends highlighted in [1]. The term Business Ecosystem was first introduced by Moore as a metaphor inspired by ecological ecosystems [2]. On the other hand, the research area of Collaborative Networks (CN) [3], which has a wider scope, classifies a business ecosystem as a subclass of the $\mathrm{CN}$ taxonomy [4]. As such, and in order to emphasize the collaborative dimension, the term Collaborative Business Ecosystem (CBE) has been adopted [5], and a CBE was modelled as an environment of agents (representing the organizations), which collaborate to accomplish business opportunities [6].

In this work it is assumed that the assessment of the collaboration benefits of a CBE, using a set of Performance Indicators (PIs) such as the ones proposed in [6] and [7], influence the behaviour of the agents inducing some degree of self-adjustment of their profile, leading to an improvement of their individual performance and of the 
CBE as a whole, promoting its sustainability. The main purpose of the paper is to present an approach to allow verifying the mentioned assumption.

The remaining sections are organized as follows: section two briefly positions this work in the context of innovation in industry and service systems; section three describes the proposed model to assess the CBE and describes the profile of organizations; section four presents one performance indicator used to illustrate the assessment; section five presents the experimental evaluation of the simulation model using some parametrized scenarios, and including a discussion of results. The last section summarizes the contributions and identifies further research directions.

\section{Relationship to Innovation in Industry and Services}

Business ecosystems have evolved with the continued increasing in digitalization and interconnection of systems, from traditional industrial sectors to digital business ecosystems supported by computer networks and collaboration platforms. This transition may entail substantial improvements for society and economy, also enabling the shift of business ecosystems towards the age of Industry 4.0, particularly in the Collaborative Industry 4.0 [8].

The essence of the Industry 4.0 concept "the leap from digital back to physical" , "a state in which manufacturing systems and the objects they create, communicate, analyse and use that information to drive further intelligent action back in the physical transition" [9], coupled with the concept of Cyber-Physical Systems (CPS), enable the implementation of more effective mechanisms to measure the performance of a $\mathrm{CBE}$ and of its individual organizations. Thus, the real time measurements to determine the performance indicators could facilitate the decision-making processes of organizations regarding selection of partners to collaborate in order to achieve more innovative, productive and value-added solutions, contributing to a more sustainable ecosystem.

\section{A Simulation Model to Evaluate the Collaboration of a CBE}

The objective of a model to evaluate collaboration within a CBE is to be able to study the evolution of the behaviour of its members, when influenced by the measurement of performance indicators, thus allowing to check how these indicators contribute (or not) to the improvement of performance and collaboration sustainability. As such, a simulation model designated by PAAM (Performance Assessment and Adjustment Model), depicted in Fig. 1, was proposed in [6]. The CBE is modelled as an environment containing a set of agents representing the Organizations $\left(\mathrm{O}_{\mathrm{i}}\right)$, which collaborate by creating Virtual Organizations $\left(\mathrm{VO}_{\mathrm{i}}\right)$ in response to market opportunities, designated as Collaboration Opportunities $\left(\mathrm{CoOp}_{\mathrm{i}}\right)$. These $\mathrm{CoOp}_{\mathrm{i}}$ are represented in the model by links between agents with weights $\mathrm{w}_{\mathrm{ij}}$, meaning the number of times an agent $\mathrm{O}_{\mathrm{i}}$ collaborated with another $\mathrm{O}_{\mathrm{j}}$.

Collaboration can be assessed using the set of performance indicators such as those proposed in [6] and [7]: the Innovation Indicator (II), to evaluate the capability of the 
organizations to create new patents, services or products; the Contribution Indicator (CI), to evaluate the value generated by organizations, creating or accepting collaboration opportunities; and the Prestige Indicator (PI), to evaluate the prominence of a particular organization over others, to participate in collaboration opportunities. It is assumed that such assessment through the given performance indicators will influence the behaviour of organizations causing their self-adjustment, trying to "look better" in face of the used metrics, resulting in an improvement of the ecosystem as a whole.

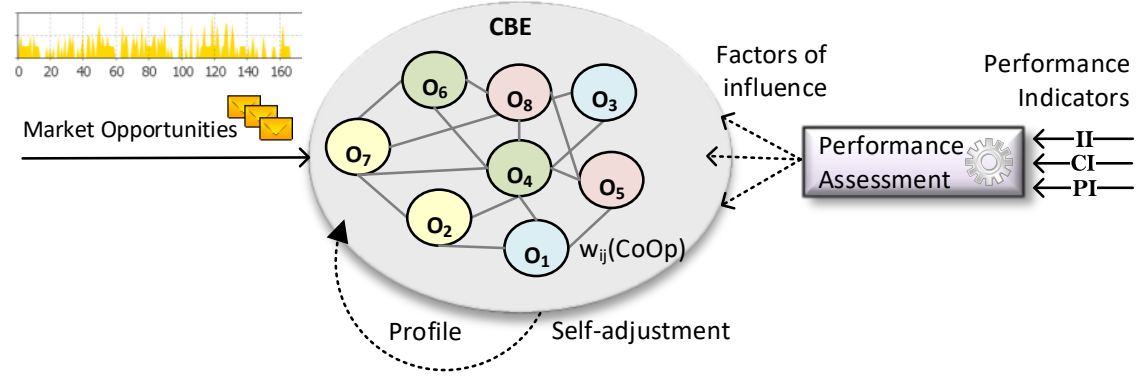

Fig. 1. PAAM (Performance Assessment and Adjustment Model) for a CBE.

The organizations are characterized by different profiles categorized into several classes of responsiveness, to better capture the diversity of a business ecosystem. In the current version of the simulation model, the following classes are considered as described in Table 1: The Social, Selfish, Innovator, and Crook. Each class expresses the way organizations collaborate (or not) in response to market opportunities, using a composition of three parameters (contact rate, acceptance rate and new products rate) with decimal values ranging from 0 to 1 , to produce different collaboration behaviour. For instance, social organizations as opposed to selfish, are more likely to contact other organizations to collaborate and are likely to accept more invitations. On the other hand, innovator organizations tend to accept more opportunities involving the creation of new patents or products. Finally, a few crook or opportunistic organizations are also considered to make the simulation model more realistic. The values in Table 1 are only for illustration and can be fine-tuned for each scenario. For each CBE a certain number of agents of each class will be considered.

Table 1. Characterization of the classes of responsiveness of organizations.

\begin{tabular}{lcccc}
\hline \multicolumn{5}{c}{ Classes of Responsiveness of Organizations } \\
\hline \multicolumn{1}{c}{ Parameters [0..1] } & Social & Selfish & Innovator & Crook \\
\hline Contact rate & 0,8 & 0,1 & 0,4 & 0,1 \\
Aceptance rate & 0,6 & 0,2 & 0,5 & 0,1 \\
New products rate & 0,2 & 0,2 & 0,9 & 0,1 \\
\hline
\end{tabular}


The importance (weight) given to each performance indicator by the CBE manager will result in a set of influence factors that will change the internal behaviour model of agents.

\section{The Contribution Indicator to Evaluate the CBE}

The Contribution Indicator (CI) is the one chosen among the performance indicators proposed in [6] to illustrate the experimental evaluation, using a simulation model of a CBE. The CI measures the contribution of the organizations to create value in the CBE using the metrics described in Table 2.

Table 2. Description of the metrics used to calculate the Contribution Indicator.

\begin{tabular}{|c|c|}
\hline \multicolumn{2}{|r|}{ Metrics of the Contribution Indicator (CI) } \\
\hline Metric & Description \\
\hline $\mathrm{O}_{1, \cdots, \mathrm{O}_{\mathrm{n}}}$ & Organizations in the $\mathrm{CBE}$ \\
\hline$\# \mathrm{O}$ & No. of organizations in the CBE \\
\hline$\# \mathrm{CoOp}_{\mathrm{i}}$ in & No. of collaboration opportunities the organization $\mathrm{O}_{\mathrm{i}}$ gained from the $\mathrm{CBE}$ \\
\hline$\# \mathrm{CoOp}$ i out & No. of collaboration opportunities the organization $\mathrm{O}_{\mathrm{i}}$ brought in the $\mathrm{CBE}$ \\
\hline
\end{tabular}

The value of $C I_{i}$ for each individual organization $O_{i}$, is calculated by the weighted indegree centrality $C_{D}\left(O_{i}\right)$ in and the weighted outdegree centrality $C_{D}\left(O_{i}\right)$ out as expressed in [10] and [11], which correspond, respectively, to the sum of the collaboration opportunities received in and sent from each organization, according to formulas (1) and (2). The resulting $C I_{i} i$ and $C I_{i}$ out are normalized values between 0 and 1 , because are divided respectively by the maximum weighted indegree centrality, $C_{D}\left(O^{*}\right)$ in , and maximum weighted outdegree centrality, $C_{D}\left(O^{*}\right)$ out .

$$
\begin{gathered}
C I_{i} \text { in }=\frac{C_{D}\left(O_{i}\right) \text { in }}{C_{D}\left(O^{*}\right) \text { in }}=\frac{\sum_{j} O_{i j} \# C o O p_{i j} \text { in }}{C_{D}\left(O^{*}\right) \text { in }} \\
C I_{i} \text { out }=\frac{C_{D}\left(O_{i}\right) \text { out }}{C_{D}\left(O^{*}\right) \text { out }}=\frac{\sum_{j} O_{i j} \# C o O p_{i j} \text { out }}{C_{D}\left(O^{*}\right) \text { out }}
\end{gathered}
$$

The value of contribution for the whole ecosystem, $C I_{C B E}$, is evaluated by $C I_{C B E} t$ and $C I_{C B E} d$. The $C I_{C B E}$, calculated by formula (3), is a ratio of the total number of collaboration opportunities created in the CBE by the total number of organizations.

$$
C I_{C B E} t=\frac{\sum_{i} \# C o O p_{i}}{\# O}
$$


The $C I_{C B E} d$, calculated by formula (4), is the centrality degree of the CBE according to [10] and [11], which gives the degree to which the most active organization exceeds the contribution of the others.

$$
C I_{C B E} d=\frac{\sum_{i}\left[C_{D}\left(O^{*}\right)-C_{D}\left(O_{i}\right)\right]}{\max \sum_{i}\left[C_{D}\left(O^{*}\right)-C_{D}\left(O_{i}\right)\right]}
$$

where $C_{D}\left(O_{i}\right)$ is the weighted indegree centrality of the organization $O_{i}, C_{D}\left(O^{*}\right)$ is the largest value of $C_{D}\left(O_{i}\right)$ for any organization in the $\mathrm{CBE}$ and $\max _{D}(C B E)$ is the maximum possible sum of differences in organization centrality for the CBE. According to [10], the maximum sum of differences is achieved for a star or a wheel graph (the most centralized graphs), which in an unweighted undirect graph with $n$ nodes, is calculated by (n-2) pairs of nodes multiplied by (n-1) maximum links. In weighted direct graphs, the maximum sum of differences is given by the maximum weighted input (maximum indegree of all organizations) multiplied by $(\# O-1)$ maximum links, allowing the reformulation of (4) resulting in formula (5).

$$
C I_{C B E} d=\frac{\sum_{i}\left[C_{D}\left(O^{*}\right) i n-C_{D}\left(O_{i}\right) i n\right]}{C_{D}\left(O^{*}\right) i n *(\# O-1)}
$$

In formula (5), the indegree is used instead of outdegree, because it better represents the importance of the organizations in the CBE. Degree-based measures of graph centrality reflects the relative dominance of a single point [10]. Thus, an organization with a high indegree, means that it was invited to collaborate by many others, which is more relevant than a high outdegree (organizations invited). Similarly, a scientific paper that is cited by many others has high relevance. The resulting $C I_{C B E} d$ is a normalized value between 0 and 1 , meaning that if $C I_{C B E} d=0$, all organizations have equal relevance, and $C I_{C B E} d=1$ if there is an organization, $\mathrm{O}^{*}$, that completely dominates the $\mathrm{CBE}$ with respect to centrality.

\section{Experimental Evaluation of the CBE}

The proposed PAAM simulation model for the experimental evaluation of a CBE, was developed with the AnyLogic Multimethod Simulation Software [12], using agent-based modelling (ABM), system dynamics (SD), and discrete elements (statecharts, events, timers, etc.).

The CBE is composed of an environment of agents (representing the organizations), which have a certain behaviour, simulated through a state-chart. Organizations are characterized by a profile classified according to the mentioned classes of responsiveness (Social, Selfish, Innovator and Crook) that define how they respond to market opportunities.

A market opportunity is also modelled as an agent including a task description, a number of resources needed to accomplish the task, a duration expressed in days and a flag indicating if it is a new product. For the current experiment, and for illustration purposes, new opportunities are generated by following discrete uniform distributions 
suitable to better simulate the randomness and diversity of market demand. When a new market opportunity is created, the number of resources follows a discrete uniform distribution, which generates values with equal probability between 1 and a parametrized maximum number of resources. The duration also follows a discrete uniform distribution between 1 and a parametrized maximum number of days. Finally, the flag indicating if it is a new product, follows a Bernoulli distribution [13] with probability $\mathrm{p}=10 \%$ of total generated market opportunities in the time window of the simulation model. This is a discrete probability distribution of any single experiment that asks a yes-no question, whose result is "yes" with probability $p$ and "no" with probability (1-p). The Bernoulli distribution is adequate since it allows to randomly generate in the $\mathrm{CBE}$, a certain percentage $(\mathrm{p})$ of new patents or products from the total number of market opportunities.

Responding to the incoming market opportunities, organizations interact creating collaboration opportunities by inviting and accepting invitations from other organizations in the CBE. The interactions are registered in the model by weighted links that represent the number of collaboration opportunities each organization received from or sent to others.

Fig. 2 illustrates the PAAM simulation model, showing a CBE environment after a period of one year, parametrized with 6 social organizations, 5 selfish, 3 innovative and 1 crook. In this example, market opportunities arrive at a rate of 1 thousand per year using the Poisson distribution [14], a discrete probability distribution quite popular for modelling the number of times an event occurs in an interval of time or space. This distribution allows to simulate a parameterized number of market opportunities, arriving randomly to the $\mathrm{CBE}$ during the time of the experiment.

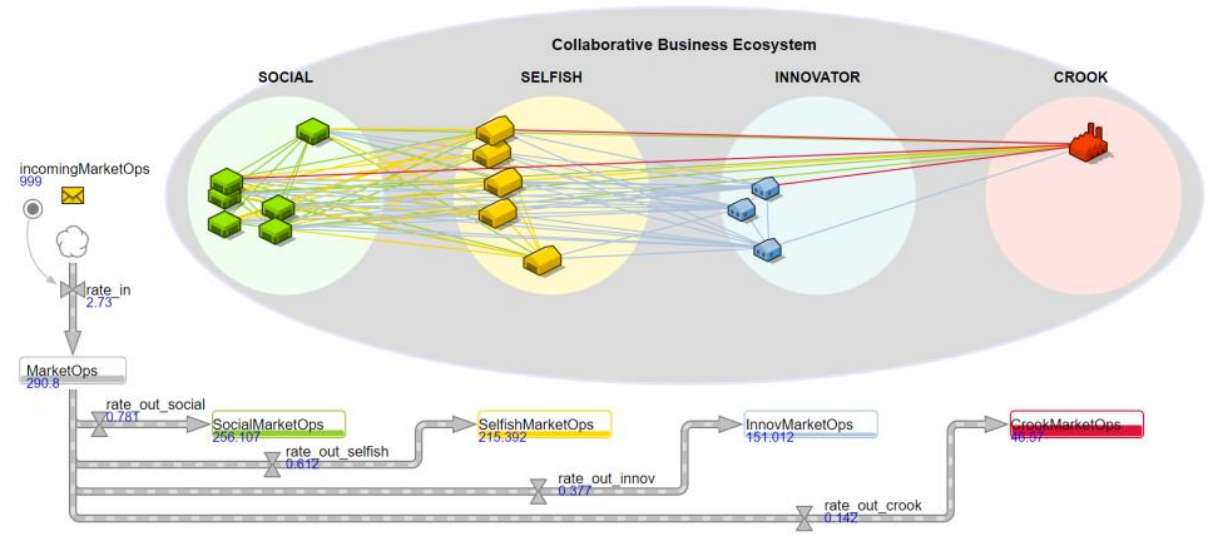

Fig. 2. PAAM simulation model, showing the CBE environment.

As mentioned, the organizations in the $\mathrm{CBE}$, are autonomous agents characterized by a profile (contact rate, acceptance rate and new products rate) according to their classes of responsiveness. Fig. 3 shows the example of a social organization whose behaviour is modelled by a state-chart and by two stocks of resources to perform the tasks associated with the incoming market opportunities. Stocks manage the flow of available and allocated resources, which after being allocated and after the execution time of tasks, are released to accept new ones. When an organization receives a new 
market opportunity, based on its class and needed resources for the task, it distributes business units inviting other organizations to collaborate, using formula (6).

$$
\text { units }_{\text {to distribute }}=\text { binomial(contactRate, } n \text { Resources) }
$$

The binomial distribution used in (6), is a discrete probability distribution with parameters $p=$ contactRate and $n=n$ Resources, which gives the number (bounded by $[0, n])$ of successes in a sequence of $\mathrm{n}$ independent experiments, each asking a yes-no question. High values of $\mathrm{p}$ give greater probability to get higher values of $\mathrm{n}$. Thus, this distribution allows the simulation of the behaviour of the agents depending on their class of responsiveness, that is, the higher the contact rate of an organization, the greater the probability of distributing more business units.

The organizations that receive the invitation, accept it or not based on the organization's class and available resources, using formula (7).

$$
\begin{aligned}
& \text { accept } t_{\text {to }_{\text {collaborate }}} \\
& =[\text { bernoulli(acceptanceRate }) \| \text { bernoulli(newProductsRate })]^{\text {\&\& availableResources }}
\end{aligned}
$$

The Bernoulli distribution [13] used in (7), is a discrete probability distribution of any single experiment that asks a yes-no question, whose result is "yes" with probability $p=$ acceptanceRate or $p=n e w P r o d u c t s R a t e$, and "no" with probability (1p). Thus, this distribution can be used to simulate the behaviour of the agents according to their class of responsiveness. The higher the acceptance rate or the new product rate of an organization, the greater the likelihood of accepting the invitation to collaborate if the required resources are available.

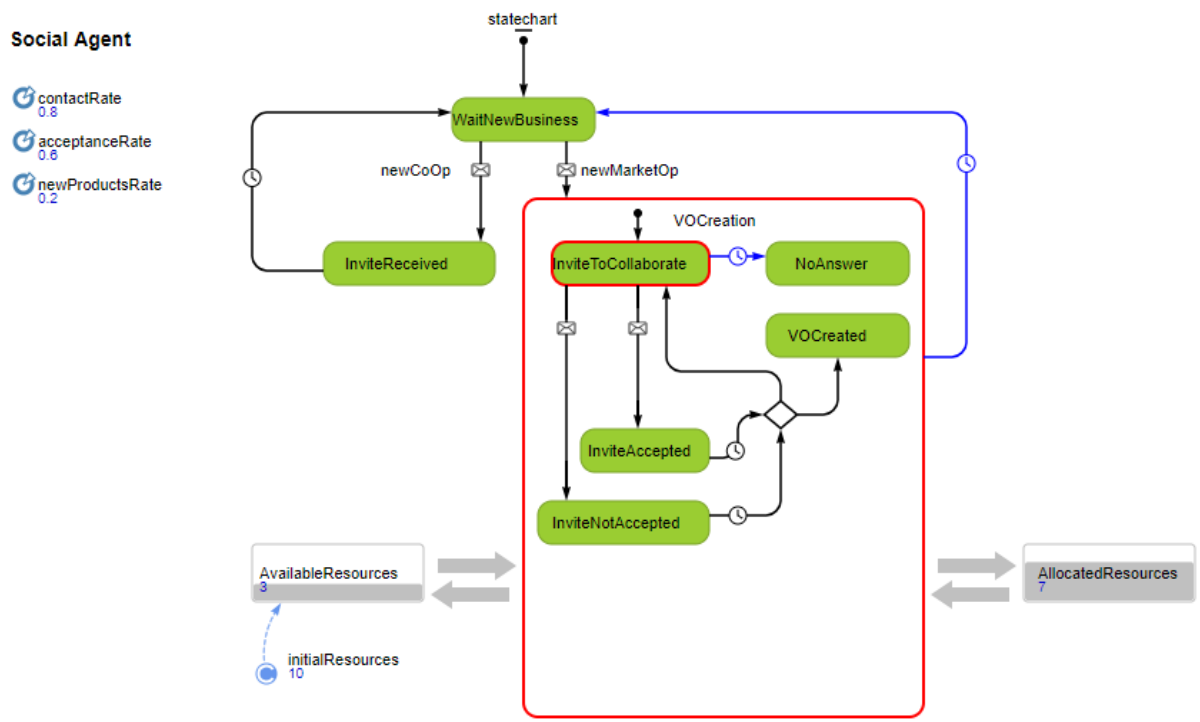

Fig. 3. Zoom-in of a social agent, showing its behaviour modelled by a state-chart and system 
dynamics.

After running the model, the $\mathrm{CI}$ is calculated for each organization and for the whole ecosystem, resulting in the values displayed in Table 3 . The column ${ } I_{i} i n$ shows the normalized value of collaboration opportunities that each organization received from others, accepting invitations to collaborate. On the other hand, the column $\mathrm{CI}_{i}$ out shows the normalized value of collaboration opportunities that each organization created in the $\mathrm{CBE}$, inviting other organizations to collaborate.

As expected, it can be verified in the results of Table 3, that the social organizations have the highest values of $\mathrm{CI}_{i}$ out due to their high contact rate. Social organizations, along with innovative organizations, also have the highest values of $C I_{i} i n$, as both have relatively high acceptance rates. In the case of innovative organizations, the acceptance of collaboration is reinforced by a high new products rate.

Finally, $C I_{C B E} t=19,5$ is the ratio of the total number of collaborations opportunities generated in the $\mathrm{CBE}$ by the total number of organizations, and $C I_{C B E} d=0,42$, the degree to which the most active organization in the CBE exceeds the number of collaboration opportunities of the others. This last value of centrality indicates that there is some polarization of the distribution of collaboration (as expected in social and innovative organizations) in the $\mathrm{CBE}$, since this value is normalized between 0 and 1 , where zero indicates an equal distribution of collaboration among all organizations. 
Table 3. Calculated values of the $\mathrm{CI}$ for each individual organization and for the whole CBE.

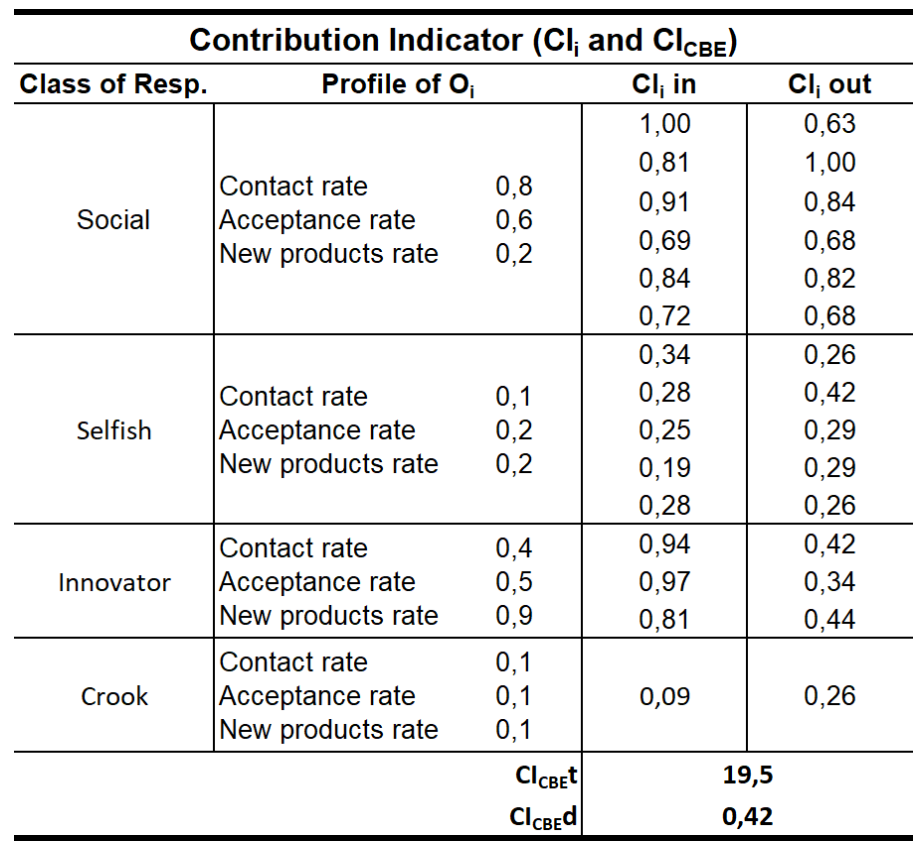

After running the simulation model again, varying the profile of social organizations, increasing their acceptance rates to 1,0 , it can be seen from the results obtained in Table 4 that collaboration increased in social organizations, also causing an increase in the ratio of collaboration $C I_{C B E} t=27,1$ as well as in the centrality $C I_{C B E} d=0,48$. 
Table 4. Calculated values of the CI after incrementing the contact and acceptance rates of social organizations.

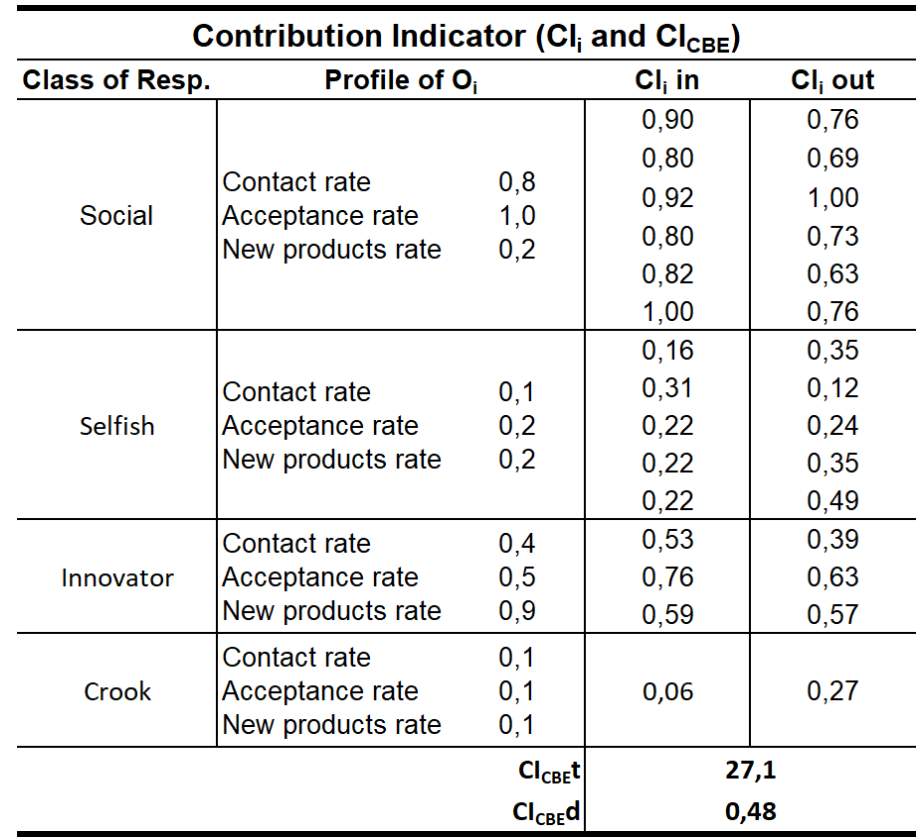

The results achieved in this work, based on the presented simulation model and scenarios, are a preliminary basis for the ongoing research. The next step is the verification of the evolution of the behaviour of a CBE, varying the weights of the PIs. It is expected that by using the PIs as a factor of influence in the profile of the organizations, they can self-adjust their behaviour, improving the performance of the CBE.

\section{Conclusions and Further Work}

The experimental evaluation in the previous section showed that a simulation model of a CBE can be defined and evaluated through the PIs proposed, more particularly, the $\mathrm{CI}$ to measure the collaboration of individual organizations and the $\mathrm{CBE}$ as a whole. It also showed that several scenarios could be modelled, varying the profile of the organizations, resulting in different measures of collaboration.

The ongoing work encompasses the calculation of the measures for the remaining PIs (II and PI), using all the indicators as a factor of influence of the behaviour of the organizations, to be possible to analyse the changes in the CBE by varying the weights of the PIs.

For future work, a more complete and dynamic simulation model can be set considering the integrating of elements of VO Performance Measurement (VOPM) proposed in the ECOLEAD project [15]. The characteristics and requirements of VOs 
establish specific performance perspectives of collaboration of their members, whose classes of responsiveness (Table 1) and behaviour (Fig. 3), used in this experimental evaluation, can be improved considering characteristics of reliability, flexibility, commitment and communication. On the other hand, the influence on the organizations of the calculated PIs, for the selection of the collaboration partners, as well as their dynamic shift from one class to another in different periods of time, are also possibilities for future work.

Finally, more diversified simulation scenarios should be defined, varying the number of organizations and tested boundary conditions.

\section{Acknowledgments}

This work benefited from the ongoing research within the CoDIS (Collaborative Networks and Distributed Industrial Systems Group) which is part of both the New University of Lisbon (UNL) - Faculty of Sciences and Technology, and the UNINOVA - CTS (Center of Technology and Systems). Partial support also comes from Fundação para a Ciência e Tecnologia through the PEST program UID/EEA/00066/2013.

\section{References}

1. M Canning and E Kelly. Business ecosystems come of age, 2015. (accessed 7 Jan 2019) https://www2.deloitte.com/content/dam/insights/us/articles/platform-strategy-new-levelbusiness-trends/DUP_1048-Business-ecosystems-come-of-age_MASTER_FINAL.pdf.

2. James F Moore. Predators and prey: a new ecology of competition. Harvard business review, 71(3):75-86, 1993.

3. Luis M Camarinha-Matos and Hamideh Afsarmanesh. Collaborative networks: a new scientific discipline. Journal of intelligent manufacturing, 16(4-5):439-452, 2005.

4. Luis M Camarinha-Matos and Hamideh Afsarmanesh. Collaborative networks: Reference modeling. Springer Science \& Business Media, 2008.

5. Paula Graça and Luis M. Camarinha-Matos. The need of performance indicators for collaborative business ecosystems. In Technological Innovation for Cloud-Based Engineering Systems, pages 22-30. Springer International Publishing, 2015.

6. Paula Graça and Luis M. Camarinha-Matos. Evolution of a collaborative business ecosystem in response to performance indicators. In Collaboration in a Data-Rich World, IFIP AICT 506, pages 629-640. Springer International Publishing, 2017.

7. Paula Graça and Luís M. Camarinha-Matos. A proposal of performance indicators for collaborative business ecosystems. In Collaboration in a Hyperconnected World, pages 253-264. Springer International Publishing, 2016.

8. Luis M. Camarinha-Matos, Rosanna Fornasiero, and Hamideh Afsarmanesh. Collaborative networks as a core enabler of industry 4.0. In Collaboration in a Data-Rich World, IFIP AICT 506, pages 3-17. Springer International Publishing, 2017.

9. Brenna Sniderman, Monika Mahto, and Mark J Cotteleer. Industry 4.0 and manufacturing ecosystems: Exploring the world of connected enterprises. Deloitte Consulting, 2016. (accessed 7 Jan 2019) https://dupress.deloitte.com/content/dam/dup-usen/articles/manufacturing-ecosystems-exploring-world-connectedenterprises/DUP_2898_Industry4.0ManufacturingEcosystems.pdf. 
10.Linton C Freeman. Centrality in social networks conceptual clarification. Social networks, 1(3):215-239, 1978.

11.Tore Opsahl, Filip Agneessens, and John Skvoretz. Node centrality in weighted networks: Generalizing degree and shortest paths. Social networks, 32(3):245-251, 2010.

12.Andrei Borshchev. The big book of simulation modeling: multimethod modeling with AnyLogic 6. 2013.

13.J. H. C. W. Introduction to mathematical probability. Science Progress (1933- ), 33(130):350-350, 1938.

14.Frank A Haight and Frank A Haight. Handbook of the Poisson distribution. 1967. New York: Jon Wiley \& Sons, 1967.

15.Luis Camarinha-Matos, Hamideh Afsarmanesh, and Martin Ollus. Methods and Tools for Collaborative Networked Organizations. Springer: New York, 2008. 